\title{
AUTOMATICITY AND COMMUTATIVE SEMIGROUPS
}

\author{
MICHAEL HOFFMANN and RICHARD M. THOMAS \\ Department of Mathematics and Computer Science, University of Leicester, Leicester LE1 7RH, England \\ e-mail:mh55@mcs.le.ac.uk,rmt@mcs.le.ac.uk
}

(Received 13 June, 2000; revised 17 November, 2000)

\begin{abstract}
We give an example of a finitely generated commutative semigroup that is not automatic.
\end{abstract}

2000 Mathematics Subject Classification. 20M05, 20M35.

1. Introduction. A natural question concerning automatic semigroups is the following: is every finitely generated commutative semigroup automatic? (This is stated as an open problem in [2].) If we restrict semigroups to groups the answer is known to be positive; a proof can be found in [4], for example.

One might expect that this result generalizes to semigroups and that all finitely generated commutative semigroups will be automatic; however, this turns out not to be the case. We will look at the commutative semigroup $Q$ defined by the presentation

$$
\begin{array}{r}
\langle a, b, x, y: \quad a a x=b x, b b y=a y, a b=b a, a x=x a, \\
a y=y a, b x=x b, b y=y b, x y=y x\rangle .
\end{array}
$$

We will prove that $Q$ is not automatic (see Example 4.1 below).

Before we do this, we will list some preliminary results from other sources in Section 2, and then prove some technical lemmas in Section 3, all of which we will need for the proof that $Q$ is not automatic which we will establish in Section 4. One of these technical results, Lemma 3.5, is a sufficient condition for a semigroup not to be automatic and this may be of some independent interest; in general, it is often difficult to establish that particular semigroups are not automatic. We finish the paper with some open questions concerning the automaticity of commutative semigroups in Section 5.

2. Preliminaries. In this section, we list some preliminary definitions and results we need and introduce some notation. For general background on semigroups see (for example) [7] or [8] and, for formal languages, see [5] or [6]. For a general account of automatic semigroups, see [2].

For any set $S$, we let $\wp(S)$ denote the set of all subsets of $S$. For any finite set $A$, we let $A^{+}$denote the set of all non-empty words over $A$, and let $A^{*}$ denote the set of all words over $A$ (including the empty word $\epsilon$ ). For any word $\alpha$ in $A^{*}$, we let $|\alpha|$ denote the length of $\alpha$ (where $|\epsilon|$ is taken to be 0 ). If $a \in A$, we let $|\alpha|_{a}$ denote the number of occurrences of $a$ in the word $\alpha$.

If $S$ is a semigroup and $A \subseteq S$ is a set of generators of $S$, then there is a natural homomorphism $\theta: A^{+} \rightarrow S$ where each word $\alpha$ in $A^{+}$is mapped to the 
corresponding element of $S$. We will normally be concerned with finite sets $A$, so that the semigroup $S$ is finitely generated. Where there is no danger of confusion, we will suppress the reference to $\theta$ by simply writing $\alpha$ for the element of the semigroup represented by $\alpha$. In this context, if $\alpha$ and $\beta$ are elements of $A^{*}$, we will write $\alpha \equiv \beta$ if $\alpha$ and $\beta$ are identical as words, and (for $\alpha, \beta \in A^{+}$) we will write $\alpha=\beta$ if $\alpha$ and $\beta$ represent the same element of $S$ (i.e. if $\alpha \theta=\beta \theta$ ). If we wish to stress which semigroup we are working in, we will write $\alpha{ }_{S} \beta$ if $\alpha$ and $\beta$ represent the same element of the semigroup $S$. We may also then write $\alpha=s$ (or $\alpha{ }_{S} s$ ), where $\alpha \in A^{+}$and $s \in S$, which says that $\alpha \theta=s$ in $S$.

As in the case of automatic groups, we will want to consider automata accepting pairs $(\alpha, \beta)$ of words with $\alpha, \beta \in A^{+}$. If $\alpha \equiv a_{1} a_{2} \ldots a_{n}$ and $\beta \equiv b_{1} b_{2} \ldots b_{m}$, this is accomplished by having an automaton with input alphabet $A \times A$ and reading pairs $\left(a_{1}, b_{1}\right),\left(a_{2}, b_{2}\right)$, and so on. To deal with the case where $n \neq m$, we introduce a padding symbol $\$$. More formally, as with automatic groups, we define a mapping $\delta_{A}: A^{*} \times A^{*} \rightarrow A(2, \$)^{*}$, where $\$ \notin A$ and $A(2, \$)=((A \cup\{\$\}) \times(A \cup\{\$\}))-\{(\$, \$)\}$, by

$$
(\alpha, \beta) \delta_{A}= \begin{cases}\left(a_{1}, b_{1}\right) \ldots\left(a_{n}, b_{n}\right) & \text { if } n=m \\ \left(a_{1}, b_{1}\right) \ldots\left(a_{n}, b_{n}\right)\left(\$, b_{n+1}\right) \ldots\left(\$, b_{m}\right) & \text { if } n<m \\ \left(a_{1}, b_{1}\right) \ldots\left(a_{m}, b_{m}\right)\left(a_{m+1}, \$\right) \ldots\left(a_{n}, \$\right) & \text { if } n>m\end{cases}
$$

Given this, we make the following definition:

Definition 2.1. If $S$ is a semigroup, $A$ is a finite set, $L$ is a regular subset of $A^{+}$, and $\phi: A^{+} \rightarrow S$ is a homomorphism with $L \phi=S$, we say that $(A, L)$ is an automatic structure for $S$ if

1. $L_{=}=\{(\alpha, \beta): \alpha, \beta \in L, \alpha=\beta\} \delta_{A}$ is a regular subset of $A(2, \$)^{*}$, and

2. $L_{a}=\{(\alpha, \beta): \alpha, \beta \in L, \alpha a=\beta\} \delta_{A}$ is a regular subset of $A(2, \$)^{*}$ for each $a \in A$. If a semigroup $S$ has an automatic structure $(A, L)$ for some $A$ and $L$, then we say that $S$ is automatic.

We next recall some standard facts about regular languages (see [1], [4] and [6] for example):

Proposition 2.2. If $A$ is a finite set and if $K$ and $L$ are regular subsets of $A^{*}$, then $K \cup L, K \cap L, K-L, K L$ and $K^{*}$ are regular.

Proposition 2.3. If $A$ and $B$ are finite sets, if $\phi: A^{*} \rightarrow B^{*}$ is a homomorphism, and if $L$ is a regular subset of $B^{*}$, then $L \phi^{-1}$ is a regular subset of $A^{*}$.

Proposition 2.4. If $A$ is a finite set and if $K$ and $L$ are regular subsets of $A^{*}$, then $(K \times L) \delta_{A}$ is a regular subset of $A(2, \$)^{*}$.

Proposition 2.5. If $A$ is a finite set and if $U_{1}, \ldots, U_{n}$ are regular languages over $A(2, \$)$, then the set

$$
\begin{gathered}
\left\{(\alpha, \beta) \delta_{A}: \alpha, \beta \in A^{*} \text { and there exist } \omega_{1}, \ldots, \omega_{n-1} \in A^{*}\right. \text { such that } \\
\left(\alpha, \omega_{1}\right) \delta_{A} \in U_{1},\left(\omega_{1}, \omega_{2}\right) \delta_{A} \in U_{2}, \ldots, \\
\left.\left(\omega_{n-2}, \omega_{n-1}\right) \delta_{A} \in U_{n-1},\left(\omega_{n-1}, \beta\right) \delta_{A} \in U_{n}\right\}
\end{gathered}
$$

is regular. 
If $L$ is a regular language over $A$, then $(L \times L) \delta_{A}$ is regular over $A(2, \$)$ by Proposition 2.4; so, if $U$ is a regular subset of $A(2, \$)^{*}$, then $U \cap(L \times L) \delta_{A}$ is regular by Proposition 2.2. So, by replacing each $U_{i}$ by $U_{i} \cap(L \times L) \delta_{A}$, we may generalize Proposition 2.5 slightly to the following result.

Proposition 2.6. If $L$ is a regular language over the set $A$ and if $U_{1}, \ldots, U_{n}$ are regular languages over $A(2, \$)$, then the set

$$
\begin{aligned}
\left\{(\alpha, \beta) \delta_{A}:\right. & \alpha, \beta \in L \text { and there exist } \omega_{1}, \ldots, \omega_{n-1} \in L \text { such that } \\
& \left(\alpha, \omega_{1}\right) \delta_{A} \in U_{1},\left(\omega_{1}, \omega_{2}\right) \delta_{A} \in U_{2}, \ldots \\
& \left.\left(\omega_{n-2}, \omega_{n-1}\right) \delta_{A} \in U_{n-1},\left(\omega_{n-1}, \beta\right) \delta_{A} \in U_{n}\right\}
\end{aligned}
$$

is regular.

We recall the definition of an "automatic structure with uniqueness":

Definition 2.7. Let $(A, L)$ be an automatic structure for $S$. We say that $(A, L)$ is an automatic structure with uniqueness for $S$ if $L$ maps one-to-one onto $S$.

We then have (as for groups):

Lemma 2.8. If $S$ is a semigroup with an automatic structure $(A, L)$, then there exists an automatic structure $(A, K)$ with uniqueness for $S$ such that $K \subseteq L$.

See [2] for a proof of Lemma 2.8. We also recall the following result from [2].

Proposition 2.9. Let $S$ be a semigroup and let $S^{1}$ be the monoid formed by adding an identity element to $S$. Then $S$ is automatic if and only if $S^{1}$ is automatic.

Lastly, we recall the following useful result from [3].

Theorem 2.10. If $M$ is an automatic monoid and if $A$ is any finite (semigroup) generating set for $M$, then there is a regular subset $L$ of $A^{+}$such that $(A, L)$ is an automatic structure for $M$.

3. Loops and sequences. We now establish some results we will need in the proof that the semigroup $Q$ mentioned in Section 1 is not automatic. The following idea is not new; it is just convenient for us to give a name to this phenomenon.

Definition 3.1. Let $L$ be regular language over a finite alphabet $A$. We call $v \in A^{+}$a loop of a word $\alpha$ in $L$ if there exist $u, w \in A^{*}$ with $\alpha \equiv u v w$ and $\{u\}\{v\}^{*}\{w\} \subseteq L$.

We call $v \in A^{+}$a reduced loop of a word $\alpha$ in $L$ if there exist $u, w \in A^{*}$ with $\alpha \equiv u v w$ and $\{u\}\{v\}^{*}\{w\} \subseteq L$ (so that $v$ is a loop) and if there do not exist $\gamma_{1} \in A^{*}$, $\gamma_{2} \in A^{+}$and $\gamma_{3} \in A^{*}$ with $\left|\gamma_{1}\right|+\left|\gamma_{3}\right| \geq 1, v \equiv \gamma_{1} \gamma_{2} \gamma_{3}$ and $\left\{u \gamma_{1}\right\}\left\{\gamma_{2}\right\}^{*}\left\{\gamma_{3} w\right\} \subseteq L$.

The point we wish to note here is the following. 
REMARK 3.2. Let $L$ be a regular language and let $M$ be a finite automaton accepting $L$. Suppose that $M$ has $n$ states. By the pumping lemma for regular languages every word $\alpha$ in $L$ of length at least $n$ contains a loop. So the set of reduced loops

$$
\left\{v \in A^{+}: v \text { is a reduced loop of some word in } L\right\}
$$

of $L$ is finite.

Having done this, we introduce the idea of a "sequence".

Definition 3.3. Let $S$ be a finitely generated semigroup and let $A$ be a finite generating set for $S$. If $a \in A$, then the maps $\sigma_{a}, \overline{\sigma_{a}}: \wp\left(A^{+}\right) \rightarrow \wp\left(A^{+}\right)$are defined as follows:

$$
\begin{aligned}
& \sigma_{a}(X)=\left\{\beta \in A^{+}: \beta=_{S} \alpha a \text { for some } \alpha \in X\right\}, \\
& \overline{\sigma_{a}}(X)=\left\{\beta \in A^{+}: \beta a=_{S} \alpha \text { for some } \alpha \in X\right\} .
\end{aligned}
$$

A map $\sigma=\tau_{1} \tau_{2} \ldots \tau_{n}$, with each $\tau_{i}$ of the form $\sigma_{a}$ or $\overline{\sigma_{a}}$ (for some $a \in A$ in each case), is called a sequence of $S$ over $A$.

Note that, by our conventions, $\sigma(X)$ denotes a subset of the semigroup $S$ as well as a subset of the free semigroup $A^{+}$, and we will use this in what follows. There is clearly no problem with doing this, as, if $\beta_{1}$ and $\beta_{2}$ are words in $A^{+}$with $\beta_{1}={ }_{S} \beta_{2}$, then $\beta_{1} \in \sigma(X)$ (as an element and a subset of $A^{+}$) if and only if $\beta_{2} \in \sigma(X)$.

Despite the fact that we have elsewhere written our maps on the right, we are writing our sequences on the left (i.e. we are writing $\sigma(X)$ as opposed to $X \sigma$ ) as this seems to be easier to read in this case. Notwithstanding this, we will still apply our map composition as if the maps were written on the right, i.e. the sequence $\tau_{1} \tau_{2} \ldots \tau_{n}$ will represent $\tau_{1}$ applied first, followed by $\tau_{2}$, and so on. In addition, when applying a sequence $\sigma$ to a singleton set $\{\alpha\}$, we will simply write $\sigma(\alpha)$ in place of $\sigma(\{\alpha\})$. We then have:

Lemma 3.4. Let $S$ be a semigroup with an automatic structure $(A, L)$ and let $\sigma$ be a sequence of $S$ over $A$. Then the set

$$
L_{\sigma}=\left\{(\alpha, \beta) \delta_{A}: \alpha, \beta \in L \text { and } \beta \in \sigma(\alpha)\right\}
$$

is regular.

Proof. For every $a \in A$ we have that the sets $L_{\sigma_{a}}=L_{a}$ and

$$
L_{\overline{\sigma_{a}}}=\left\{(\alpha, \beta) \delta_{A}:(\beta, \alpha) \delta_{A} \in L_{a}\right\}
$$

are regular. The sequence $\sigma$ can be written as $\sigma=\tau_{1} \tau_{2} \ldots \tau_{n}$ with each $\tau_{i}$ of the form $\sigma_{a}$ or $\overline{\sigma_{a}}$. So 


$$
\begin{aligned}
L_{\sigma}= & \left\{(\alpha, \beta) \delta_{A}: \alpha, \beta \in L \text { and } \beta \in \sigma(\alpha)\right\} \\
= & \left\{(\alpha, \beta) \delta_{A}: \text { there exists } \gamma \in L \text { with }(\alpha, \gamma) \delta_{A} \in L_{\tau_{1}}\right. \text { and } \\
& \left.\quad(\gamma, \beta) \delta_{A} \in L_{\tau_{2} \ldots \tau_{n}}\right\} \\
\ldots & \\
= & \left\{(\alpha, \beta) \delta_{A}: \text { there exists } \gamma_{1}, \ldots, \gamma_{n-1} \in L \text { with }\left(\alpha, \gamma_{1}\right) \delta_{A} \in L_{\tau_{1}},\right. \\
& \left.\quad\left(\gamma_{1}, \gamma_{2}\right) \delta_{A} \in L_{\tau_{2}}, \ldots\left(\gamma_{n-2}, \gamma_{n-1}\right) \delta_{A} \in L_{\tau_{n-1}},\left(\gamma_{n-1}, \beta\right) \delta_{A} \in L_{\tau_{n}}\right\}
\end{aligned}
$$

is regular by Proposition 2.6. Note that we are using the fact that $L$ maps onto $S$ in asserting that we may choose the words $\gamma_{1}, \ldots, \gamma_{n-1}$ to lie in $L$.

The following result is really the critical one in proving that our commutative semigroup $Q$ from Section 1 is not automatic.

Lemma 3.5. Let $S$ be a semigroup and let $A$ be a finite generating set for $S$. Suppose that $B$ is an infinite regular subset of $A^{+}$such that no word in $B$ represents the same element of $S$ as any other word in $A^{+}$. Let $\sigma$ be a sequence of $S$ over $A$ such that

(i) $\sigma(\beta) \cap B$ is a finite subset of $S$ for all $\beta \in B$, and

(ii) if $B^{\prime} \subseteq B$ is infinite then $\left\{|\gamma|-|\beta|: \beta \in B^{\prime}, \gamma \in \sigma(\beta) \cap B\right\}$ is not bounded.

Then $S$ is not automatic.

Proof. Suppose that $S$ is automatic, so that $S^{1}$ is automatic by Proposition 2.9. Let $A_{1}=A \cup\{e\}$ where $e$ represents the identity element of $S^{1}$. By Lemma 2.8 and Theorem 2.10 there exists an automatic structure $\left(A_{1}, L\right)$ with uniqueness for $S^{1}$.

Let $\phi: A_{1}^{*} \rightarrow A^{*}$ be the homomorphism defined by

$$
a \phi=a \text { for } a \in A ; e \phi=\epsilon .
$$

Note that, if $\alpha \phi \not \equiv \epsilon$, then both $\alpha$ and $\alpha \phi$ represent an element of $S$ (i.e. they do not represent the adjoined identity), and, moreover, they both represent the same element of $S$. So $\alpha$ and $\alpha^{\prime}$ represent the same element of $S$ if and only if $\alpha \phi$ and $\alpha^{\prime} \phi$ represent the same element of $S$. Given this, if $X \subseteq A^{+}$, then we have

$$
\sigma_{a}(X \phi)=\sigma_{a}(X) \text { and } \overline{\sigma_{a}}(X \phi) \subseteq \overline{\sigma_{a}}(X)
$$

for $a \in A$. Note that, in $\sigma_{a}(X)$, we are considering $\sigma_{a}$ as a sequence for $S^{1}$ over $A_{1}$ and $\sigma_{a}(X)$ as a subset of $S^{1}$, and, in $\sigma_{a}(X \phi)$, we are considering $\sigma_{a}$ as a sequence for $S$ over $A$ and $\sigma_{a}(X \phi)$ as a subset of $S$ (and similarly for $\overline{\sigma_{a}}(X)$ and $\overline{\sigma_{a}}(X \phi)$ ). The point is that, if $X \subseteq A^{+}$, then $\sigma_{a}(X)$ cannot contain $e$; on the other hand, $\overline{\sigma_{a}}(X)$ might contain $e$, in which case $\overline{\sigma_{a}}(X \phi)=\overline{\sigma_{a}}(X)-\{e\}$.

Let $C=B \phi^{-1} \cap L$. If $\beta \in B$, then $\beta$ represents some element $s$ of $S$. Choose $\alpha$ to be the unique element of $L$ representing $s$. Since $\alpha$ and $\alpha \phi$ both represent $s$ and $\beta$ is the unique element of $A^{+}$representing $s$ by hypothesis, we must have that $\alpha \phi \equiv \beta$. So $\phi$ maps $C$ bijectively onto $B$. In addition, using Propositions 2.2 and 2.3 and the fact that $B$ is regular, we see that $C$ is regular. Let $n$ be the number of states in a finite state automaton accepting $C$. 
Let $P$ be the set of reduced loops of words in $C$. By Remark 3.2 we have that $P$ is finite. Let $v \in P$ be such that $l=\frac{|v|}{|v \phi|}$ is minimal, i.e. such that there exists no $v^{\prime} \in P$ with

$$
\frac{\left|v^{\prime}\right|}{\left|v^{\prime} \phi\right|}<\frac{|v|}{|v \phi|} .
$$

Because $v$ is a loop in $C$, there exist $u, w \in A_{1}^{*}$ such that $\{u\}\{v\}^{*}\{w\} \subseteq C$ with $|u|<n$ and $|w|<n$. Let $D=\{u\}\{v\}^{*}\{w\}$. Let $\eta \in D$, say $\eta \equiv u v^{i} w$. Then

$$
\begin{aligned}
|\eta| & =|u|+|w|+i|v| \\
& =|u|+|w|+i|v \phi| l \\
& =|u|+|w|+(|\eta \phi|-|u \phi|-|w \phi|) l \\
& \leq|u|+|w|+|\eta \phi| l \\
& \leq 2 n-2+|\eta \phi| l .
\end{aligned}
$$

Let $\gamma \in C$. If $|\gamma| \geq n$ then $\gamma$ contains a reduced loop $v^{(1)}$ in $C$, say $\gamma \equiv u^{(1)} v^{(1)} w^{(1)}$. Let $\gamma^{(1)} \equiv u^{(1)} w^{(1)}$, so that $\gamma^{(1)} \in C$. Then

$$
\begin{aligned}
|\gamma| & =\left|\gamma^{(1)}\right|+\left|v^{(1)}\right| \\
& =\left|\gamma^{(1)}\right|+\frac{\left|v^{(1)}\right|}{\left|v^{(1)} \phi\right|}\left|v^{(1)} \phi\right| \\
& \geq\left|\gamma^{(1)}\right|+\left|v^{(1)} \phi\right| l .
\end{aligned}
$$

Iterating, we get

$$
\begin{aligned}
|\gamma| & \geq\left|\gamma^{(1)}\right|+\left|v^{(1)} \phi\right| l \\
& \geq\left|\gamma^{(2)}\right|+\left|v^{(1)} \phi\right| l+\left|v^{(2)} \phi\right| l \\
& \geq\left|\gamma^{(3)}\right|+\left|v^{(1)} \phi\right| l+\left|v^{(2)} \phi\right| l+\left|v^{(3)} \phi\right| l \\
& \ldots \\
& \geq\left|\gamma^{(r)}\right|+\left|v^{(1)} \phi\right| l+\left|v^{(2)} \phi\right| l+\left|v^{(3)} \phi\right| l+\ldots+\left|v^{(r)} \phi\right| l \\
& =\left|\gamma^{(r)}\right|+\left(|\gamma \phi|-\left|\gamma^{(r)} \phi\right|\right) l,
\end{aligned}
$$

where $\left|\gamma^{(r)}\right|<n$, and so $\left|\gamma^{(r)} \phi\right|<n$. Hence

$$
\begin{aligned}
|\gamma| & \geq\left|\gamma^{(r)}\right|+\left(|\gamma \phi|-\left|\gamma^{(r)} \phi\right|\right) l \\
& \geq|\gamma \phi| l-\left|\gamma^{(r)} \phi\right| l \\
& >|\gamma \phi| l-n l .
\end{aligned}
$$

Now we consider $|\gamma|-|\eta|$ for $\eta \in D$ and $\gamma \in \sigma(\eta) \cap C$. We have, from equations (2) and (3),

$$
\begin{aligned}
|\gamma|-|\eta| & >|\gamma \phi| l-n l-2 n+2-|\eta \phi| l \\
& =-n l-2 n+2+(|\gamma \phi|-|\eta \phi|) l .
\end{aligned}
$$


Let $B^{\prime}=D \phi \subseteq C \phi=B$, so that $B^{\prime}$ is infinite (as $\phi$ maps $C$ bijectively onto $B$ ); see Figure 1.

Now the set

$$
\left\{\left|\gamma^{\prime}\right|-\left|\eta^{\prime}\right|: \eta^{\prime} \in B^{\prime}, \gamma^{\prime} \in \sigma\left(\eta^{\prime}\right) \cap B\right\}
$$

is not bounded by hypothesis (ii). Since $\phi$ maps $D$ onto $B^{\prime}$, we have that the set

$$
\left\{\left|\gamma^{\prime}\right|-|\eta \phi|: \eta \in D, \gamma^{\prime} \in \sigma(\eta \phi) \cap B\right\}
$$

is not bounded. As $\phi$ maps $C$ bijectively onto $B$, we have that

$$
\{|\gamma \phi|-|\eta \phi|: \eta \in D, \gamma \in C, \gamma \phi \in \sigma(\eta \phi) \cap B\}
$$

is not bounded. Since $\gamma \in C$, the condition $\gamma \phi \in B$ is superfluous, and we have that

$$
\{|\gamma \phi|-|\eta \phi|: \eta \in D, \gamma \in C, \gamma \phi \in \sigma(\eta \phi)\}
$$

is not bounded. Since $\gamma \phi$ represents the same element of $S$ as $\gamma$, and as $\sigma(\eta \phi) \subseteq \sigma(\eta)$ by (1), we have that

$$
\{|\gamma \phi|-|\eta \phi|: \eta \in D, \gamma \in C, \gamma \in \sigma(\eta)\}
$$

is not bounded; note that we are now considering $\sigma$ as a sequence in $S^{1}$. Using (4), we see that

$$
\{|\gamma|-|\eta|: \eta \in D, \gamma \in \sigma(\eta) \cap C\}
$$

is not bounded. Let

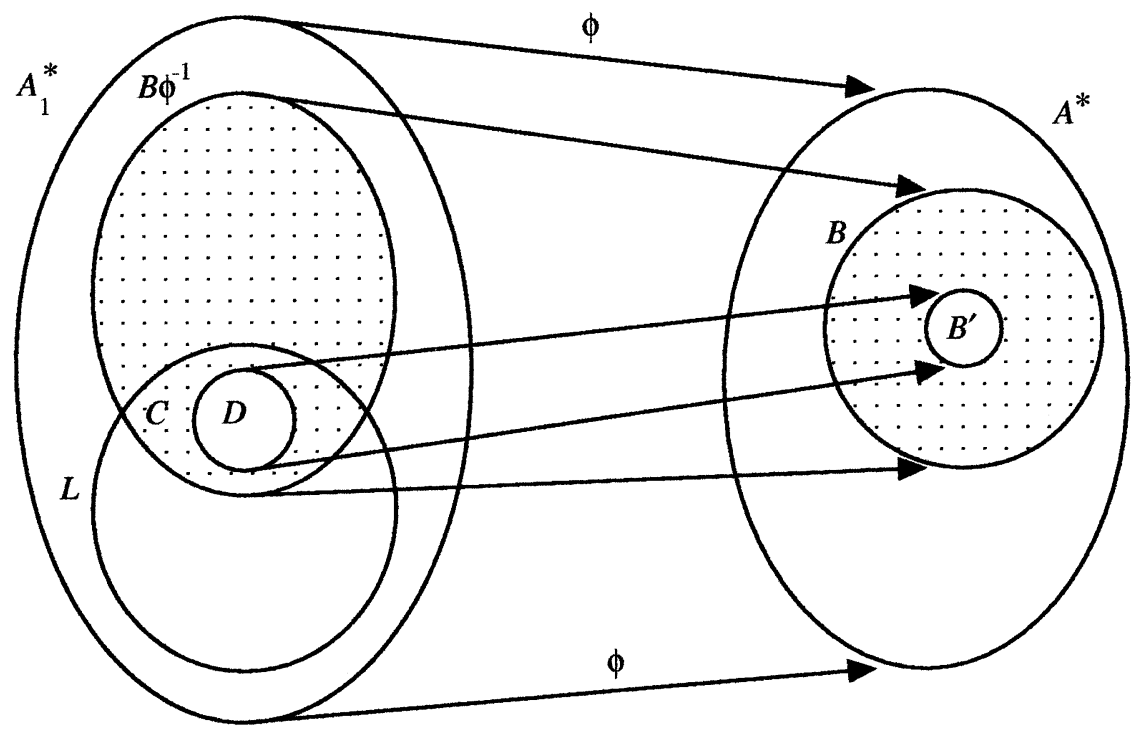

Figure 1. Mappings. 


$$
\begin{aligned}
E & =\left\{(\eta, \gamma) \delta_{A_{1}}: \eta \in D, \gamma \in \sigma(\eta) \cap C\right\} \\
& =(D \times C) \delta_{A_{1}} \cap L_{\sigma},
\end{aligned}
$$

where the notation $L_{\sigma}$ is as in Lemma 3.4.

The set $E$ is the intersection of two regular languages by Proposition 2.4 and Lemma 3.4, and is therefore also regular by Proposition 2.2. Let $m$ be the number of states in a finite state automaton $M$ accepting $E$. Let $\eta \in D$ and $\gamma \in \sigma(\eta) \cap C$ with $|\gamma|-|\eta| \geq m$. So $(\eta, \gamma) \delta_{A_{1}} \in E$ and the last $m+1$ letters of $(\eta, \gamma) \delta_{A_{1}}$ are of the form $\left(\$, a_{i}\right)$ for some $a_{i} \in A_{1}$. Whilst reading the last $m+1$ letters of $(\eta, \gamma) \delta_{A_{1}}$ the machine $M$ must be in the same state $q$ twice. We can decompose $\gamma$ as $\gamma_{1} \gamma_{2} \gamma_{3}$, where $\gamma_{2}$ is the loop read between successive such visits to $q$. We can pump this loop $\gamma_{2}$ in $M$ and get that $\left(\eta, \gamma_{1} \gamma_{2}^{i} \gamma_{3}\right) \delta_{A_{1}} \in E$ for all $i \geq 0$. So $\gamma_{1} \gamma_{2}^{i} \gamma_{3} \in \sigma(\eta) \cap C$, and then $\left(\gamma_{1} \gamma_{2}^{i} \gamma_{3}\right) \phi \in \sigma(\eta \phi) \cap B$ for all $i \geq 0$.

Recall that $L$ has uniqueness and that $\phi$ maps $C$ bijectively to $B$; so $\left(\gamma_{1} \gamma_{2}^{i} \gamma_{3}\right) \phi \neq_{S}\left(\gamma_{1} \gamma_{2}^{j} \gamma_{3}\right) \phi$ for $i \neq j$. Hence $\sigma(\eta \phi) \cap B$ is not finite, which contradicts hypothesis (i).

4. Commutative but not automatic. We can now demonstrate the existence of a finitely generated commutative semigroup (namely our example $Q$ from Section 1) that is not automatic.

Example 4.1. The commutative semigroup $Q$ defined by the presentation

$$
\begin{array}{r}
\wp=\langle a, b, x, y: a a x=b x, b b y=a y, a b=b a, a x=x a, \\
a y=y a, b x=x b, b y=y b, x y=y x\rangle
\end{array}
$$

is not automatic.

To see this, we let $A=\{a, b, x, y\}$ and $B=\left\{a^{i}: i>0\right\}$. None of the relations in $\wp$ can be applied to an element of $B$. Therefore, for $\alpha \in A^{+}$with $\alpha={ }_{Q} \beta$ for some $\beta \in B$, we must have that $\alpha \equiv \beta$. Let $\sigma=\sigma_{y} \overline{\sigma_{y}} \sigma_{x} \overline{\sigma_{x}}$ (recalling that our convention is that we then apply $\sigma_{y}$ first).

First we note that, if $\eta \in\{a, b\}^{+}$, then

$$
\begin{aligned}
& \zeta \in \sigma_{x}(\eta) \Leftrightarrow|\zeta|_{x}=1 \text { and }|\eta|_{a}+2|\eta|_{b}=|\zeta|_{a}+2|\zeta|_{b}, \\
& \zeta \in \sigma_{y}(\eta) \Leftrightarrow|\zeta|_{y}=1 \text { and } 2|\eta|_{a}+|\eta|_{b}=2|\zeta|_{a}+|\zeta|_{b} .
\end{aligned}
$$

Combining these facts together, we see that

$$
\begin{aligned}
\zeta \in \sigma(\eta) \Leftrightarrow & \text { there exists } \mu \in\{a, b\}^{+} \text {with } \\
& 2|\eta|_{a}+|\eta|_{b}=2|\mu|_{a}+|\mu|_{b} \text { and }|\mu|_{a}+2|\mu|_{b}=|\zeta|_{a}+2|\zeta|_{b} \\
\Leftrightarrow & \text { there exists } \mu \in\{a, b\}^{+} \text {with } \\
& 4|\eta|_{a}+2|\eta|_{b}=4|\mu|_{a}+2|\mu|_{b}=3|\mu|_{a}+|\zeta|_{a}+2|\zeta|_{b} .
\end{aligned}
$$

Letting $k$ denote $|\mu|_{a}$, we have that

$$
\sigma\left(a^{i}\right)=\left\{\zeta \in\{a, b\}^{+}: 4 i=3 k+|\zeta|_{a}+2|\zeta|_{b} \text { for some } k \in \mathbb{N}\right\}
$$


and so

$$
\sigma\left(a^{i}\right) \cap B=\left\{a^{j}: \text { there exists } k \in \mathbb{N} \text { such that } j=4 i-3 k\right\}
$$

Therefore $\sigma(\beta) \cap B$ is a finite subset of $Q$ for all $\beta \in B$.

We also see from (5) that $a^{4 i} \in \sigma\left(a^{i}\right) \cap B$ for all $i>0$. Hence, for any infinite $B^{\prime} \subseteq B$, we have that

$$
\left\{|\gamma|-|\beta|: \beta \in B^{\prime}, \gamma \in \sigma(\beta) \cap B\right\}
$$

is not bounded. So, by Lemma 3.5, we have that $Q$ is not automatic.

5. Questions. We have seen that a finitely generated commutative semigroup need not be automatic. However, it may well be that there are some natural extra assumptions that could be added to ensure automaticity.

One natural choice would be that of "bounded indegree".

Definition 5.1. A semigroup $S$ generated by a finite set $A$ is said to have bounded indegree if there exists a constant $k$ such that, for all $s \in S$, the set $\{t \in S: s=t a$ for some $a \in A\}$ has size at most $k$. choose.

Note that this property does not depend on which (finite) generating set we

Proposition 5.2. If a semigroup $S$ has bounded indegree with respect to one finite generating set $A$, then it has bounded indegree with respect to any finite generating set.

Proof. Assume that $S$ has bounded indegree with respect to $A$, i.e. that there is a constant $k$ such that, for any $a \in A$, we have

$$
|\{t \in S: s=t a\}| \leq k
$$

for all $s \in S$. So, if $\alpha \in A^{+}$, then

$$
|\{t \in S: s=t \alpha\}| \leq k^{|\alpha|}
$$

for all $s \in S$.

Let $B$ be another finite generating set for $S$. We can express every element $b$ of $B$ by a word $\alpha_{b}$ of $A^{+}$. Let $p=\max \left\{\left|\alpha_{b}\right|: b \in B\right\}$; note that $B$ is finite and therefore $p$ exists. So, if $b \in B$, then

$$
\{t \in S: s=t b\}=\left\{t \in S: s=t \alpha_{b}\right\}
$$

has size at most $k^{p}$ for all $s \in S$.

The example of a commutative semigroup $Q$ given above which is not automatic has unbounded indegree as all the elements $a^{i}(i \in \mathbb{N})$ are distinct and $a^{4^{i}} x y=a x y$ for all $i \in \mathbb{N}$. So we have the following question. 
Question 5.3. Is every finitely generated commutative semigroup that has bounded indegree automatic?

Recall that Green's relations $\mathcal{R}$ and $\mathcal{L}$ are defined on a semigroup $S$ by $a \mathcal{R} b$ (respectively $a \mathcal{L} b$ ) if $a$ and $b$ generate the same principal right (respectively left) ideal, i.e. if and only if $a S^{1}=b S^{1}$ (respectively $S^{1} a=S^{1} b$ ). The relation $\mathcal{H}$ is the intersection of $\mathcal{R}$ and $\mathcal{L}$. The commutative semigroup $Q$ in Example 4.1 has infinitely many $\mathcal{H}$-classes (equivalently, infinitely many $\mathcal{R}$-classes or $\mathcal{L}$-classes, since the relations $\mathcal{R}, \mathcal{L}$ and $\mathcal{H}$ coincide in a commutative semigroup), and so we ask

QUESTION 5.4. Is every finitely generated commutative semigroup that has finitely many $\mathcal{H}$-classes automatic?

ACKNOWLedgements. The authors are grateful to Nik Ruškuc for several helpful conversations about the material in this paper; they would also like to thank the referee for his/her careful reading of the paper and for a useful observation about the exposition. The authors would also like to thank Chen-Hui Chiu and Hilary Craig for all their help and encouragement.

\section{REFERENCES}

1. G. Baumslag, S. M. Gersten, M. Shapiro and H. Short, Automatic groups and amalgams, J. Pure Appl. Algebra 76 (1991), 229-316.

2. C. M. Campbell, E. F. Robertson, N. Ruškuc and R. M. Thomas, Automatic semigroups, Theoret. Comput. Sci. 250 (2001), 365-391.

3. A. J. Duncan, E. F. Robertson and N. Ruškuc, Automatic monoids and change of generators, Math. Proc. Cambridge Philos. Soc. 127 (1999), 403-409.

4. D. B. A. Epstein, J. W. Cannon, D. F. Holt, S. Levy, M. S. Paterson and W. Thurston, Word processing in groups (Jones and Barlett, 1992).

5. M. A. Harrison. Introduction to formal language theory (Addison Wesley, 1978).

6. J. E. Hopcroft and J. D. Ullmann, Introduction to automata theory, languages, and computation (Addison-Wesley, 1979).

7. J. M. Howie, Fundamentals of semigroup theory (Oxford University Press, 1995).

8. G. Lallement, Semigroups and combinatorial applications (John Wiley, 1979). 